\title{
As transformações do saber científico ao saber ensinado: o caso do logaritmo
}

\section{The transformation of scientific knowledge into taught knowledge: the case of logarithms}

\author{
Saddo Ag Almouloud ${ }^{1}$
}

\begin{abstract}
RESUMO
Para ensinar uma noção científica em um dado nível de escolaridade, é necessário que ela seja acessível aos alunos. Portanto, precisa-se transformá-la a partir de um saber de referência que é, em geral, o saber dos especialistas da disciplina (o saber sábio). Neste trabalho, são apresentadas ferramentas teóricas da didática da matemática para estudar as transformações do saber científico em saber ensinado. É tomado como objeto matemático de estudo o "logaritmo". A função logaritmo é uma das noções mais importantes das que integram o currículo do Ensino Médio. Ela tem várias aplicações em diversas áreas de conhecimento tais como: física, química, economia, astronomia, o que justifica sua manutenção nas propostas curriculares de vários países.

Palavras-chave: transposição didática; função logaritmo; ensino-aprendizagem; currículo.
\end{abstract}

\begin{abstract}
In order to teach a scientific concept at a given school level, this concept must be accessible to the students; therefore, it must be transformed based on a reference knowledge, which is, in general, the knowledge from the discipline's specialists (scholar knowledge). In this paper, theoretical tools
\end{abstract}

1 Doutorado em Educação Matemática - Universidade de Rennes I - França. Professor da Pontifícia Universidade Católica de São Paulo - PUC/SP, Brasil, saddoag@gmail.com ou saddoag@ pucsp.br 
from the didactic of Mathematics are presented to study the transformation of scientific knowledge into taught knowledge. The mathematical object on focus is the "logarithm." The logarithm function is one of the most important concepts in the High School curriculum. It has various applications in a wide range of knowledge areas, such as physics, chemistry, economics, astronomy, justifying its continued inclusion in the curricula proposals of various countries.

Keywords: didactic transposition; logarithm function; teaching-learning; curriculum.

\section{Introdução}

Para Guy Brousseau (1986),

um processo de aprendizagem pode ser caracterizado de modo geral (se não determinado) por um conjunto de situações identificáveis (naturais ou didáticas) reprodutíveis, conduzindo frequentemente à modificação de um conjunto de comportamentos de alunos, modificação característica da aquisição de um determinado conjunto de conhecimentos (BROUSSEAU, 1986, p. 6, tradução nossa).

Em didática da matemática procura-se teorizar os fenômenos ligados à atividade matemática, visando à especificidade do conhecimento ensinado. Para isso, o sistema mínimo a levar em consideração é o sistema didático stricto sensu (Figura 1), ou seja, as interações entre professor e alunos mediadas pelo saber nas situações do ensino. 


\section{Polopsicológico}

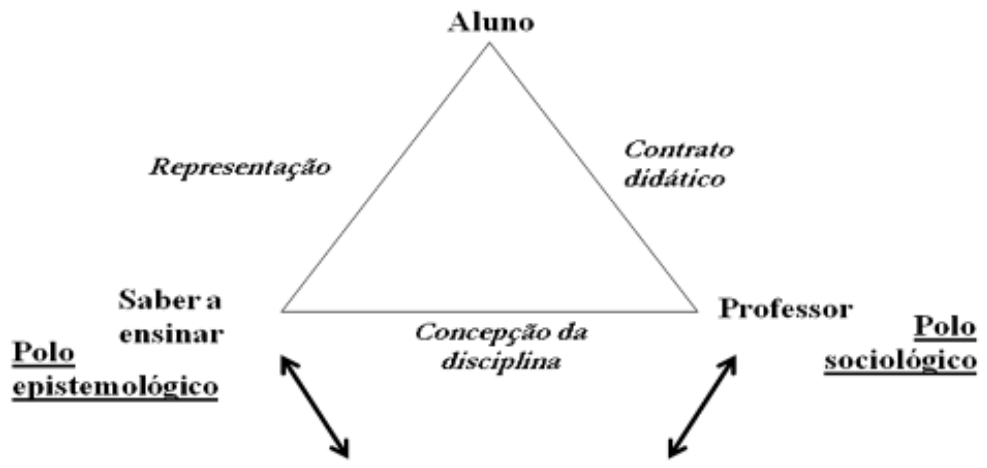

SABER DE REFERENCIA AMBIENTE

FIGURA 1 - TRIÂNGULO DIDÁTICO

Para ensinar uma noção científica em um dado nível de escolaridade, é necessário torná-la acessível aos alunos. Portanto, precisa-se transformá-la a partir de um saber de referência que, em geral, é o saber dos especialistas da disciplina (o saber sábio). Neste trabalho, apresentamos ferramentas teóricas da didática da Matemática para responder as seguintes questões: Como analisar o currículo de um nível de ensino? Para uma dada noção, quais aspectos são privilegiados no ensino, nas propostas curriculares e nas práticas de classes? Quais são os aspectos importantes da noção que estão ausentes nos processos de transformação? Quais escolhas didáticas podem ser feitas? Tomaremos como objeto matemático de estudo o "logaritmo". A função logaritmo é uma das noções mais importantes dentre as que integram o currículo do Ensino Médio. Ela tem várias aplicações em diversas áreas de conhecimento tais como a física, a química, a economia, a astronomia, o que justifica sua manutenção nas propostas curriculares de vários países.

\section{Origem do conceito de transposição didática}

Verret (1975) introduziu o conceito de transposição didática. Interessouse pela ação humana que visa à transmissão de saberes, tornando-os prontos para que sejam "ensináveis" e aprendidos. Segundo esse ponto de vista, con- 
vém, sem dúvida, tornar os saberes acessíveis aos aprendizes, mediante uma simplificação e uma vulgarização levando em conta a idade desses aprendizes e seus conhecimentos prévios. A transposição didática passa, segundo Verret, por transformações radicais. Ele aponta cinco:

- A estruturação do saber em campos e domínios distintos. Os saberes científicos já são organizados em disciplinas.

- A despersonalização do saber, ou seja, o saber sábio não está atrelado a indivíduos e grupos de indivíduos que o produzem ou o usam.

- Uma programação necessária, pois um saber a ensinar não pode ser assimilado em uma só vez; o saber ensinado passa então por caminhos de formatação balizada.

- Uma publicação do saber, em livros/revistas e propostas curriculares, que permitam a cada sujeito conhecer o saber a ensinar e as competências e habilidades a alcançar.

- Um controle das aquisições.

$\mathrm{Na}$ escola, essas transformações começam com a transposição externa (transformação de saberes e práticas em propostas curriculares) e prosseguem pela efetivação das propostas (transposição interna). Essas transformações levam em consideração as condições de trabalho do professor e do aluno na escola.

\section{Transposição didática segundo Chevallard}

O termo "transposição didática", segundo Chevallard \& Joshua (1991), designa o conjunto das transformações que sofre um saber dito sábio, para ser ensinado. $\mathrm{Ou}$ seja, refere-se às transformações que sofrem as teorias dos matemáticos quando se tornam saberes escolares, em primeiro lugar nas propostas curriculares, depois nos livros didáticos e em sala de aula. O saber sábio é construído e faz parte do patrimônio cultural do pesquisador. A sociedade solicita o ensino de uma parte desse saber por razões puramente sociais: formação profissional, por necessidades econômicas. É necessário então transformar esses saberes para que possam ser ensinados e, consequentemente, entendidos em dado nível. É, então, indispensável examinar as características do objeto de saber do ponto de vista epistemológico e das hipóteses de 
aprendizagem escolhidas:

- A construção do saber pelo pesquisador

Todo pesquisador deve por meio de uma publicação em uma revista científica tornar acessível a todos os resultados de sua pesquisa. Para isso, ele deve transformar toda a história de seus trabalhos. Ele elimina todas as reflexões inúteis, os erros, as estratégias utilizadas e que não deram certo ou que não tinham importância na resolução do problema. Além disso, ele não apresenta suas próprias motivações nem sua concepção da ciência. Ele despersonaliza o saber que comunica. Elimina também toda a história passada desse saber; e, eventualmente, não o relaciona com o problema particular que queria resolver inicialmente.

Ele desconstextualiza e destemporaliza o saber comunicado. Define previamente o vocabulário novo na forma de definição, que só é compreendida pelo leitor que possui os conhecimentos indispensáveis para assimilar o novo saber, objeto da comunicação. Ele contribui para o enriquecimento da língua matemática, trazendo um vocabulário específico. Outros pesquisadores retomarão esse novo saber para aplicá-lo na resolução de problemas. Nesta ocasião, eles o transformarão, o generalizarão em caso de necessidade.

- O ensino de um saber

O trabalho do professor supõe evidentemente um conhecimento do objeto de saber, mas também do modo pelo qual os alunos construam seus conhecimentos.

Em didática da matemática, considera-se que a aprendizagem ideal consiste em colocar o aluno em situações problemáticas cuja solução levaria à construção do conhecimento visado. O conhecimento é então recontextualizado, é conhecimento que aparece então como solução a um problema específico. Além do mais, esse novo conhecimento sendo construído pelo aluno, por sua iniciativa própria, é repersonalizado. Esta recontextualização e repersonalização constituem o trabalho do professor. Não se trata de reconstituir a origem histórica da descoberta desse saber, bem como das dificuldades que, possivelmente, o acompanharam, mas criar um caminho mais curto para o aluno partir da construção de seus conhecimentos. O trabalho do professor seria semelhante ao inverso do trabalho do pesquisador. O professor deve construir situações-problema em que o conhecimento matemático apontado seja recontextualizado e repersonalizado em vista de se tornar um conhecimento do aluno, ou seja, uma resposta mais natural às condições indispensáveis para que esse conhecimento tenha um sentido. Para Brousseau (1986), há três condições para atingir esse objetivo:

- simular, na sala de aula, uma "microssociedade" matemática para provocar um debate científico, dominando as situações de formulação e de validação;

- institucionalizar o saber cultural e comunicável que se queira ensinar 
aos alunos, pois estes também devem descontextualizar e recontextualizar seu saber.

- Construção da ferramenta matemática

Para que a ferramenta matemática construída pelo aluno se torne um objeto de saber é necessário fazê-la funcionar na resolução de outros tipos de problemas. Depois, descontextualizá-la e despersonalizá-la a fim de que ela seja reinvestida em diferentes situações (profissionais, por exemplo).

Esse trabalho de desconstextualização e de despersonalização deve necessariamente ser feito pelo aluno para que possa integrar esse novo conhecimento ao seu patrimônio cultural. Ele deve então, como o pesquisador, fazer uma comunicação escrita ao conjunto de seus colegas de classe no intuito de provocar debates que enriqueceriam, esclareceriam esse novo saber ou delimitariam as condições de suas aplicações. $\mathrm{O}$ trabalho do aluno deve ser do mesmo tipo que o do pesquisador.

- As condições concretas de ensino

As transformações do objeto de saber em objeto de ensino devem ser necessariamente acompanhadas de uma análise epistemológica, das hipóteses de aprendizagem e do contexto social. O professor não transforma por iniciativa própria o saber sábio em objeto de ensino. A escolha dos objetos a ensinar é definida institucionalmente por meio de propostas curriculares, e é controlada de alguma forma pela sociedade (autoridades locais, pais de alunos, autoridades administrativas da educação).

Na Figura 2, está representada a ideia de que o professor não tem influência direta na elaboração das propostas curriculares oficiais.

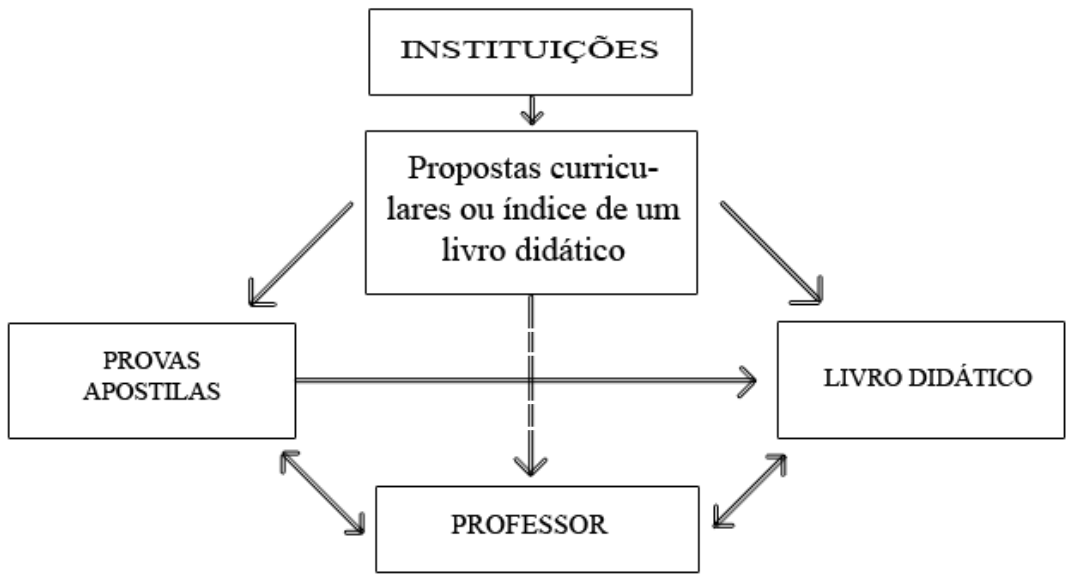


Temos um conjunto de influências de que depende a ação do professor, o exemplo mais marcante é o do papel dos livros didáticos nos processos de ensino e aprendizagem. O trabalho de transposição anterior ao trabalho do professor leva a decompor a transposição em dois níveis, conforme a Figura 3 ilustra:

\section{Saber da instituiçăo de referência}
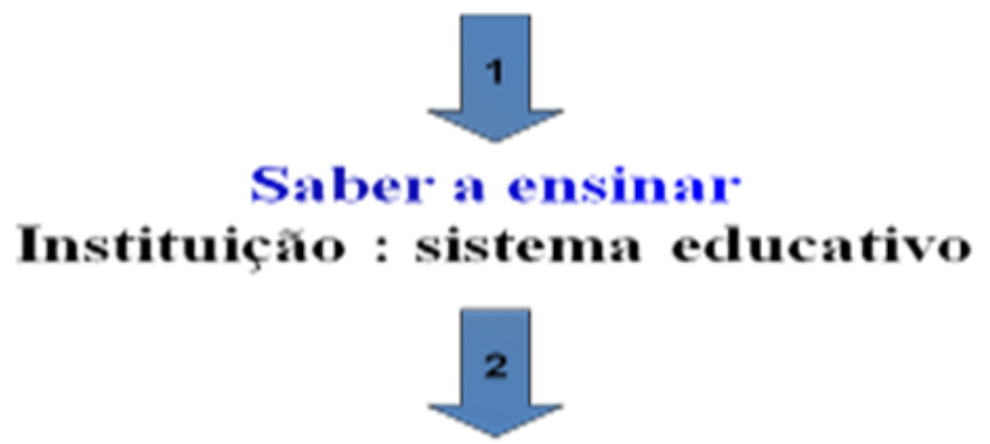

\section{Saber ensinado \\ Instituiçāo: classe}

FIGURA 3 - ETAPAS DA TRANSPOSIÇÃO DIDÁTICA

Geralmente, o professor só intervém no nível do saber ensinado. O saber a ensinar não se limita às propostas curriculares, seu ensino necessita de sua interpretação. O saber a ensinar é o que o professor acha que deve ensinar a partir da leitura de livros didáticos, do livro do professor, ou a partir de práticas tidas anteriormente. $\mathrm{O}$ texto do saber a ensinar não está completamente escrito em lugar algum. É indispensável examinar se a distância, a deformação entre o objeto de saber e o objeto de ensino não é, na pior das hipóteses, uma linguagem pseudocientífica.

As transformações do saber científico ao saber ensinado: o caso do logaritmo

A noção de logaritmo é uma das noções mais importantes que encontramos em matemática e no currículo de ensino médio. Como assinalado antes, ela tem várias aplicações em diversas áreas de conhecimento. Essas aplicações lhes con- 
ferem um papel importante no ensino. Antes de Néper (1765), o fundamento da teoria dos logaritmos era relacionar uma sequência de potências de um número, desde a época paleobabilônica.

O matemático John Néper (1614, apud GROUPE MATH, 1991) inventou a palavra e o conceito de logaritmo em 1614. Seu objetivo era simplificar o cálculo de um produto por o de uma soma. Na época, a noção de função não existia e os logaritmos serviram de ferramenta para o desenvolvimento desse conceito definido por Euler somente em 1748. Tornou-se um meio para fazer corresponder um logaritmo a cada número positivo, tratava-se então de uma função. Para Néper, os logaritmos são números que correspondem a números proporcionais. Usa uma proporção geométrica, ou seja, (a, b, c, d) são em proporção geométrica se, e somente $\mathrm{ad}=\mathrm{bc}$, assim, a soma $\log (\mathrm{a})+\log (\mathrm{d})=$ $\log (b)+\log (c)$.

Segundo Jacques Ozanam (1670, apud GROUPE MATH, 1991), “os logaritmos são números em proporção aritmética, que correspondem a outros números em proporção geométrica". Ele escolheu uma função f contínua e estritamente crescente em $I R$ * que verifica a seguinte propriedade "logarítmica": $f$ transforma toda proporção geométrica em uma proporção aritmética e tal que $f(10)=1 f(1)=0$.

\section{Um breve estudo da transposição didática nos livros didáticos}

Em alguns livros didáticos do Brasil, $\log _{a} x$ (logaritmo base $a$ de $\left.x\right)$ é $o$ número a que temos de elevar a para obter $x$, ou seja, $\log _{a} x=y \Leftrightarrow \mathrm{a}^{\mathrm{y}}=\mathrm{x}$ :

a) Existe uma função logarítmica que é inversível e cuja inversa é a função exponencial definida para todo número real.

b) Na maioria dos livros didáticos de Ensino Médio da França e dos países africanos de língua francesa, o logaritmo neperiano é definido por $\ln (t)=\int_{1}^{t} \frac{1}{x} d x d x$. Se admitirmos o seguinte teorema "Toda função $f$ contínua em um intervalo I tem primitivas", então, demonstra-se que existe uma única função F que verifica para e, portanto a função $\mathrm{f}$ definida por $\mathrm{f}(\mathrm{x})=$ tem uma primitiva $\mathrm{F}$ em tal que $\mathrm{F}(1)=0$.

Esta problemática de busca de primitivas está na origem da introdução do conceito de logaritmo. Esta concepção não pode ser ensinada no terceiro ano do Ensino Médio. A problemática de cálculo, ou seja, a relação entre multiplicação e adição, está na origem de nossa proposta, servindo-nos das proporções geométricas e aritméticas como ferramenta. Este tipo de abordagem, originada 
pelo uso da régua de cálculo, parece bem afastado do uso das calculadoras, mas guarda seu interesse na medida em que coloca em evidência uma prática de cálculo que desempenhou um papel importante na história da matemática. Nossa proposta está apoiada no uso de uma propriedade do conjunto dos números reais, mais especificamente, a do isomorfismo do grupo aditivo do conjunto dos números reais sobre o grupo multiplicativo do conjunto dos números reais estritamente positivos.

$$
\begin{aligned}
& f:(\mathbb{R},+) \rightarrow\left(I R_{+}^{*}, \times\right) \\
& f(a+b)=f(a) \times f(b)
\end{aligned}
$$

Percebemos que a maioria dos livros didáticos da França e dos países africanos de língua francesa apresenta situações em que se procuram funções que têm a seguinte propriedade:

$$
f: I R_{+}^{*} \rightarrow I R \text {, tal que } f(x y)=f(x)+f(y) .
$$

O objetivo de nossa proposta para introduzir o conceito de logaritmo é prevalecer-se da propriedade logarítmica de uma função estritamente contínua no conjunto dos números reais positivos e não nulos: "f transforma toda proporção geométrica em uma proporção aritmética". Do ponto de vista da transposição didática, há uma diferença notável entre as abordagens propostas nos livros didáticos brasileiros analisados, e as propostas da maioria dos livros didáticos franceses e da África de língua francesa. Como destacamos, os livros de língua francesa (França e África de língua francesa) definem a função logaritmo neperiano como a função $F$, primitiva da função fdefinida por $\mathrm{f} x \mapsto f(x)=\frac{1}{x}$ $\mathrm{x}$, ou seja, $\mathrm{F}(\mathrm{x})=\ln \mathrm{x}=\int_{1}^{x} \frac{d t}{t}$ sendo $\mathrm{F}(1)=0$ sendo $\mathrm{F}(1)=0$; nos livros brasileiros, o logaritmo é definido com o número y tal que $\log _{a} x=y \Leftrightarrow \mathrm{a}^{\mathrm{y}}=\mathrm{x}$.

Propomos outra maneira de introduzir e estudar a noção de função logaritmo, utilizando-se da propriedade acima citada, a partir de uma situação que permite estudar todas as propriedades dessa função, resolver equações logarítmicas, esboçar a curva e a reta tangente em um ponto da curva dessa função. Apoiamo-nos no trabalho proposto pelo Groupe MATH, IREM de Paris VII (1991). Adaptamos a situação proposta por este grupo de pesquisa. 


\section{Enunciado da situação-problema estudada ${ }^{2}$}

Apresentamos duas definições importantes para a resolução da situação:

1. Quatro números reais positivos e não nulos $a, b, c$, d dizem-se em proporção geométrica se, e somente se $\mathbf{a d}=\mathbf{b c}$.

2. Quatro números reais $a, b, c, d$ são em proporção aritmética se, $e$ somente se $\mathrm{a}+\mathrm{d}=\mathrm{b}+\mathrm{c}$.

Questão 1: Seja f uma função em $\mathrm{R}+*$. Mostre que, se f transforma toda proporção geométrica em uma proporção aritmética, então f transforma toda sequência geométrica em uma sequência aritmética (pode-se verificar que se $\left(u_{n}\right)$ é uma sequência geométrica de razão $\mathbf{q} \neq \mathbf{0}$, então $\mathbf{1}, \mathbf{q}, \mathbf{u}_{\mathbf{n}}, \mathbf{u}_{\mathbf{n + 1}}$ são em proporção geométrica).

Agora queremos traçar a curva representativa de uma função f contínua e estritamente crescente em $I R_{+}^{*}$ que verifica a seguinte propriedade "logarítmica": f transforma toda proporção geométrica em uma proporção aritmética. Para fazer essa representação, estudaremos algumas das características desta função.

Como J. Ozanam, escolhemos estudar a função $f$ tal que $\mathrm{f}(1)=0$ e $\mathrm{f}(10)=1$. Agora que tem essas informações responda às seguintes questões:

Questão 1: Calcule :

$$
f\left(100, \quad f(1000) f(10.000) \quad f\left(10^{n}\right)\right.
$$

Questão 2: a) Mostre que $f(15)=f(3)+f(5)$,

e que, para $x$ e y elementos de $R+* f(x y)=f(x)+f(y)$. função de $f(x)$.

b) Calcule, para $x$ elemento de $R+*$, o valor de $f\left(\frac{1}{x}\right)$ e $f(\sqrt{x})$ em

c) Determine, para a e b elementos de $\mathrm{R}+* \mathbf{f}(\sqrt{\mathbf{a b}})$ em função de $f(a)$ e $f(b)$.

Questão 3: Resolva no R+* as equações
a) $f(x)=1 / 2$
b) $f(x)=1 / 4$
c) $\mathrm{f}(\mathrm{x})=5 / 4$

2 Uma situação-problema é a escolha de questões abertas ou fechadas numa situação mais ou menos matematizada, envolvendo um campo de problemas colocados em um ou em vários campos de conhecimentos matemáticos. A função principal de uma situação-problema é a utilização implícita, depois explícita, de novas ferramentas matemáticas (em nosso caso, as propriedades da função f), por meio de questões que o aluno se coloca no momento de sua pesquisa. 
Questão 4: Complete a seguinte tabela:

\begin{tabular}{|l|l|l|l|l|l|l|l|l|l|l|l|l|}
\hline$x$ & $1 / 10$ & & & & 1 & & $\sqrt{\mathbf{1 0}}$ & & 10 & & & \\
\hline$f(x)$ & -1 & $-3 / 4$ & $-1 / 2$ & $-1 / 4$ & 0 & $1 / 4$ & $1 / 2$ & $3 / 4$ & 1 & $5 / 4$ & $3 / 2$ & $7 / 4$ \\
\hline
\end{tabular}

Questão 6: Em um sistema cartesiano ortogonal de unidade $2 \mathrm{~cm}$, trace a curva representativa da função $f$.

Questão 7: Considere os pontos $M_{i}$ da curva de ordenadas -1, -3/4, 1/-2, $-1 / 4,0,1 / 2,7 / 4$

a. Represente os pontos $M_{i}$ ' de coordenadas $\left(0 ; \mathrm{y}_{\mathrm{M}_{\mathrm{i}}}-0,43\right)$. Cuidado A unidade é $2 \mathrm{~cm}$.

b. Construa as retas $M_{i} \stackrel{\leftrightarrow}{M_{i}}{ }^{\prime}$.

c. Identifique a reta $M_{i} \stackrel{\leftrightarrow}{M}{ }_{i}{ }^{\prime}$ que aproxima melhor a curva. Qual é o coeficiente angular dessa reta? Encontra $\mathrm{f}(\mathrm{x})$.

\section{Análise matemática da situação-problema}

Definimos as noções de proporção geométrica e de proporção aritmética. Estas duas noções são fundamentais na resolução da situação, assim como na utilização da propriedade logarítmica da função $\mathrm{f}$.

A primeira questão tem por objetivo demonstrar que a função $f$ transforma toda progressão geométrica em uma progressão aritmética. Partindo de uma progressão geométrica $\left(\mathrm{u}_{\mathrm{n}}\right)$ de razão $\mathrm{q}$, temos que $1, \mathrm{q}$, $u_{n}, u_{n+1}$ estão em proporção geométrica, pois $1 . u_{n+1}=q u_{n}$. Logo, $p_{g}\left(1, q, u_{n}, u_{n+1}\right) \Rightarrow f(1)+f\left(u_{n+1}\right)=f(q)+f\left(u_{n}\right)$. Seja $t_{n}=f\left(u_{n}\right)$ e $t_{n+1}=f\left(u_{n+1}\right)$, portanto $t_{n+1}=r+t_{n}$, onde $r=f(q)-f(1)$.

Na segunda questão, pede-se calcular as imagens de certos números por f, levando em consideração as seguintes condições $\left\{\begin{array}{l}f(1)=0 \\ f(10)=1\end{array}\right.$. Na resolução dos diferentes itens desta, precisam ser utilizadas proporções geométricas e a propriedade algorítmica da função $\mathrm{f}$.

a) Calcule $f(100) ; f(1000) ; f(10.000)$ e $f\left(10^{\mathrm{n}}\right)$

$$
\begin{aligned}
& p_{g}(1,10,10,100) \Leftrightarrow f(100)=f(10)-f(1)+f(10)=2 f(10)=2 \\
& p_{g}(1,10,100,1000) \Leftrightarrow f(1000)=f(10)-f(1)+f(100)=1+2=3 \\
& p_{g}(1,10,1000,10000) \Leftrightarrow f(10000)=f(10)-f(1)+f(1000)=1+3=4
\end{aligned}
$$

Por indução completa, demonstra-se que $\forall n \in I N ; f\left(10^{n}\right)=n$

Para $n=0, f\left(10^{0}\right)=f(1)=0$. 
Supõe-se que $\forall n \in I N ; f\left(10^{n}\right)=n$, e demonstra-se que $f\left(10^{n+1}\right)=n+1$. $p_{g}\left(1,10,10^{n}, 10^{n+1}\right) \Leftrightarrow f\left(10^{n+l}\right)=f(10)-f(1)+f\left(10^{n}\right)=1+n$

Portanto, para todo $n \in I N, f\left(10^{n}\right)=n$ (princípio da indução completa)

b) $\alpha$ ) Demonstrar que $f(15)=f(3)+f(5)$

$p_{\mathrm{g}}(1,3,5,15) \Leftrightarrow f(10)=f(3)-f(1)+f(5)=f(3)+f(5)$, pois, $f(1)=0$ Demonstrar que para todos x e y de $I R_{+}^{*}$, tem-se $\left.f x y\right)=f(x)+f(y)$ $\mathrm{p}_{\mathrm{g}}(1, x, y, x y) \Leftrightarrow f(x y)=f(x)-f(1)+f(y)=f(x)+f(y)$ $f(x y)=f(x)+f(y)$ (Propriedade fundamental de $\mathrm{f}$ ) $\beta$ ) Deduza para todo $\mathrm{x}$ de $I R_{+}^{*}, f\left(\frac{1}{x}\right)$ e $f(\sqrt{x})$ em função de $f(x)$ $f(1)=f\left(x \frac{1}{x}\right)=f(x)+f\left(\frac{1}{x}\right)=o \Leftrightarrow f\left(\frac{1}{x}\right)=-f(x)$
$f(x)=f(\sqrt{x} \sqrt{x})=f(\sqrt{x})+f(\sqrt{x})=2 f(\sqrt{x}) \Leftrightarrow f(\sqrt{x})=\frac{1}{2} f(x)$ $\gamma)$ Calcule, para todo a e b de $I R_{+}^{*}, f(\sqrt{a b})$ em função de $f(a)$ e $f(b)$ $f(\sqrt{a b})=f(\sqrt{a} \sqrt{b})=f(\sqrt{a})+f(\sqrt{b})=\frac{1}{2} f(a)+\frac{1}{2} f(b)=\frac{1}{2}(f(a)+f(b)$. A terceira questão tem por objetivo esboçar o gráfico da curva da função f. 1.

\begin{tabular}{|c|l|l|l|l|l|l|l|l|l|l|}
\hline$x$ & $10^{-1}$ & $10^{-\frac{3}{4}}$ & $10^{-\frac{1}{2}}$ & $10^{-\frac{1}{4}}$ & 1 & $\sqrt{10}$ & $10^{\frac{3}{4}}$ & 10 & $10^{\frac{5}{4}}$ & $10^{\frac{3}{2}}$ \\
\hline$f(x)$ & -1 & $-\frac{3}{4}$ & $-\frac{1}{2}$ & $-\frac{1}{4}$ & 0 & $\frac{1}{2}$ & $\frac{3}{4}$ & 1 & $\frac{5}{4}$ & $\frac{3}{2}$ \\
\hline
\end{tabular}


2. Curva representativa de $\mathrm{f}$

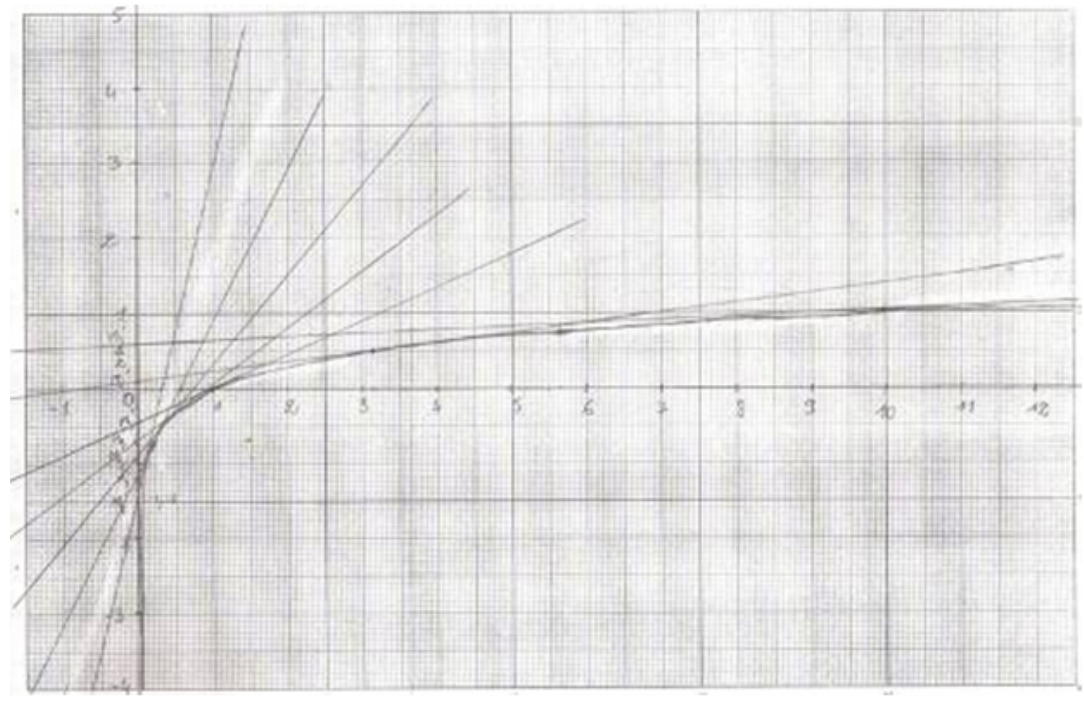

FIGURA 4 - GRÁFICO DA FUNÇÃO F E DAS RETAS M $\mathrm{M}_{\mathrm{i}}{ }^{\prime}{ }_{\mathrm{i}}$

a) $\mathrm{M}_{1}^{\prime}(0 ;-1,43) ; \mathrm{M}_{2}^{\prime}(0 ;-1,18) ; \mathrm{M}_{3}^{\prime}(0 ;-0,93) ; \mathrm{M}_{4}^{\prime}(0 ;-0,68) ; \mathrm{M}_{5}{ }_{5}(0 ;-0,43)$; $\mathrm{M}_{6}^{\prime}(0 ;-0,07) ; \mathrm{M}_{7}^{\prime}(0 ;-0,57) ; \mathrm{M}_{8}^{\prime}(0 ;-1,07)$.

b) cf. Figura 4

c) A reta que melhor aproxima a curva de fé a reta que passa pelos pontos. $M^{\prime}(0 ; 1,07)$ e $M\left(10^{\frac{3}{2}} ; \frac{3}{2}\right)$. Seu coeficiente angular é igual a $m=\frac{y_{M}-y_{M^{\prime}}}{x_{M}-x_{M^{\prime}}}=\frac{\frac{3}{2}-1,07}{10^{\frac{3}{2}}}=\frac{0,43}{10^{\frac{3}{2}}}=0,0136$

2. Análise didática da situação-problema

A situação-problema foi elaborada levando em consideração as seguintes condições:

1. Os alunos compreendem facilmente os dados e podem engajar-se na exploração desses dados com os conhecimentos disponíveis. Podem conceber claramente o que é uma resposta possível e pertinente à questão colocada.

2. A situação-problema envolve um campo conceitual (o campo conceitual do logaritmo) que desejamos efetivamente explorar e em que 
se situam as aprendizagens visadas.

3. Os conhecimentos antigos dos alunos são insuficientes para a resolução imediata do problema.

4. Os conhecimentos, objetos da aprendizagem, fornecem as ferramentas adequadas para obter a solução.

5. A situação-problema envolve os seguintes quadros: algébrico, geométrico, numérico.

$\mathrm{O}$ fato de supor que a função f é definida em $I R_{+}^{*}$ remonta à história dos logaritmos. A função f é contínua e estritamente crescente. Esta propriedade garante a unicidade das soluções das equações logarítmicas. Sabe-se que uma função contínua e crescente em intervalo é bijetora. Portanto, a equação $f(x)=m, m \in I R$ e $x \in I R_{+}^{*}$ admite uma solução única, o que permite completar a tabela.

As igualdades $f(1)=0$ e $\mathrm{f}(10)=1$ são duas condições relacionadas com a propriedade da função estudada. Os números 1 e 10 são particularmente importantes na escolha das proporções geométricas cujas imagens por f permitem resolver praticamente todas as questões da situação.

Para justificar a escolha de 0,43 , usa-se a equação da tangente à curva de $\mathrm{f}$ em um ponto $\mathrm{x}_{0}$, ou seja, $\mathrm{y}=\mathrm{f}^{\prime}\left(\mathrm{x}_{0}\right)\left(\mathrm{x}-\mathrm{x}_{0}\right)+\mathrm{f}\left(\mathrm{x}_{0}\right)$. Se $\mathrm{f}(\mathrm{x})=\log (\mathrm{x})$, então $f(x)=\frac{\ln x}{\ln 10}$ e $f^{\prime}(x)=\frac{1}{x \ln 10}$, a equação da tangente $(\mathrm{T})$ à curva de $\mathrm{f}$ em um ponto de abscissa $\mathrm{x}_{0}$ é $(\mathrm{T}): y=\frac{1}{x_{0} \ln 10}\left(x-x_{0}\right)+f\left(x_{0}\right)$, se $\mathrm{x}=0$, tem-se , $y=-\frac{1}{\ln 10}+f\left(x_{0}\right)$, mas $\frac{1}{\ln 10}=0,43$ portanto, $y=y_{m o}-0,43$, onde $\mathrm{y}_{\mathrm{M}_{0}}=\mathrm{f}\left(\mathrm{x}_{0}\right)$.

Quais conhecimentos os alunos podem mobilizar na resolução da situação?

Dizemos que um aluno possui um conhecimento matemático se ele for capaz de utilizá-lo de forma explícita na resolução de problemas. Na situação proposta, o aluno precisa minimamente mobilizar os seguintes conhecimentos: a prova por indução completa que permite demonstrar que $f\left(10^{n}\right)=n$; a propriedade logarítmica da função $f$ e as caracterizações das progressões geométricas e aritméticas que permitem desvendar as pistas que levariam à solução da situação proposta; as propriedades da raiz quadrada de um número positivo que são imprescindíveis ao cálculo $f(\sqrt{x})$ e $f(\sqrt{a b})$.

Notamos a importância e o papel das mudanças de quadro no tratamento das questões da situação. Essas mudanças permitem mudar de ponto de vista e traduzir algumas das questões da situação do quadro (DOUADY, 1986) algébrico ao quadro da geometria analítica com a finalidade específica de mobilizar as ferramentas adequadas 
à resolução dessas questões. As interações entre esses quadros constituem um poderoso instrumento para a construção, por parte do aluno, do conhecimento/saber em jogo na situação-problema a partir de seus conhecimentos disponíveis.

\section{Uma breve análise da fase experimental}

A experiência que relatamos foi realizada em uma turma de 3. ${ }^{\text {a }}$ série do Ensino Médio (Ciências Exatas) de Mali. Esta experiência foi realizada por Siaka Konaté (2006). Participaram da pesquisa da situação-problema 38 alunos que ainda não estudaram o conceito de logaritmo. Os alunos trabalharam em grupo: 7 grupos de quatro alunos e 2 grupos de 5 alunos, que denominamos de $\mathrm{G}_{1}, \mathrm{G}_{2}, \mathrm{G}_{3}, \mathrm{G}_{4}, \mathrm{G}_{5}, \mathrm{G}_{6}$, $\mathrm{G}_{7}$ (4 alunos); $\mathrm{G}_{8}$ e $\mathrm{G}_{9}$ (compostos por 5 alunos). $\mathrm{O}$ tempo previsto para a resolução da situação foi de $01 \mathrm{~h} 50$.

Na maioria dos grupos, houve confusão entre a proporção aritmética e a progressão aritmética, exceto no $\mathrm{G} 3$, que justificou sua resposta da seguinte forma: $\mathrm{v}_{\mathrm{n}+1}$ $=\mathrm{f}\left(\mathrm{u}_{\mathrm{n}+1}\right) ; \mathrm{f}(\mathrm{q})-\mathrm{f}(1)=\mathrm{r}$ et $\mathrm{f}\left(\mathrm{u}_{\mathrm{n}}\right)=\mathrm{v}_{\mathrm{n}}$, portanto $\mathrm{v}_{\mathrm{n}+1}=\mathrm{v}_{\mathrm{n}}+\mathrm{r}$.

Para o cálculo $\mathrm{f}(100)$, sabendo que $\mathrm{f}(1)=0$ e $\mathrm{f}(10)=1$, foi constatado que os alunos não sabiam por onde começar a solução do problema. Precisou-se indagá-los sobre a possibilidade de encontrar um número $\mathrm{x}$ tal que os números 1,10 , x e 100 estivessem em proporção geométrica. Esta pergunta permitiu aos alunos iniciar a resolução da questão. Para o cálculo de $\mathrm{f}\left(10^{\mathrm{n}}\right)$, a maioria dos grupos fez uma conjectura, mas sem demonstrá-la. Para ilustrar este fato, é apresentada, a seguir (Figura 5 e Figura 6), a produção do grupo $\mathrm{G}_{8}$.

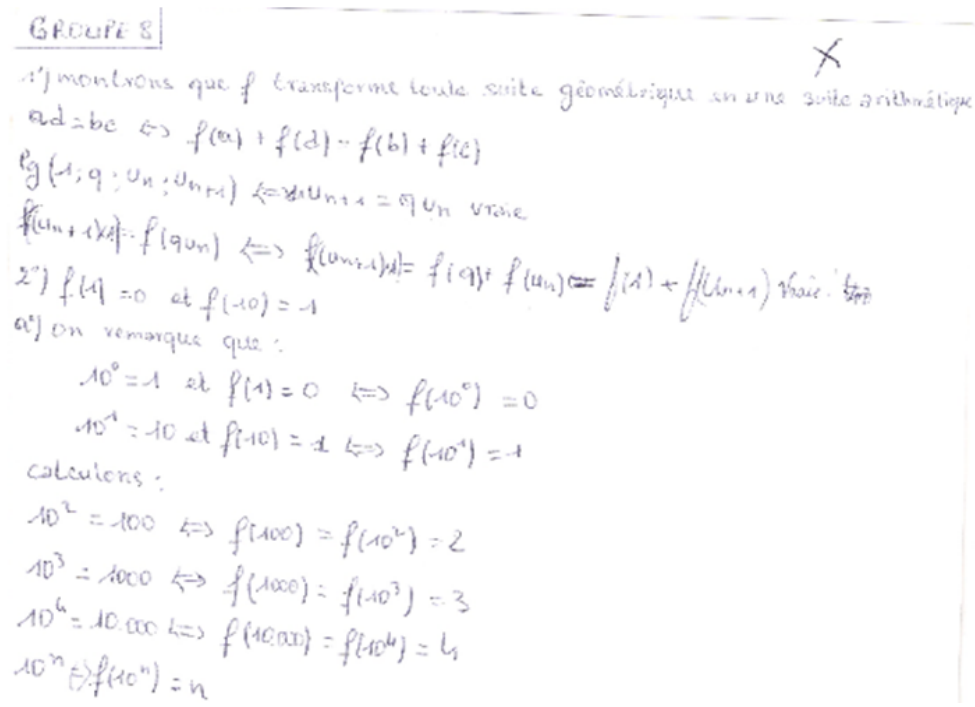

FIGURA 5 - RESOLUÇÃO APRESENTADA PELO GRUPO 8 (KONATÉ, 2006, p. 60). 


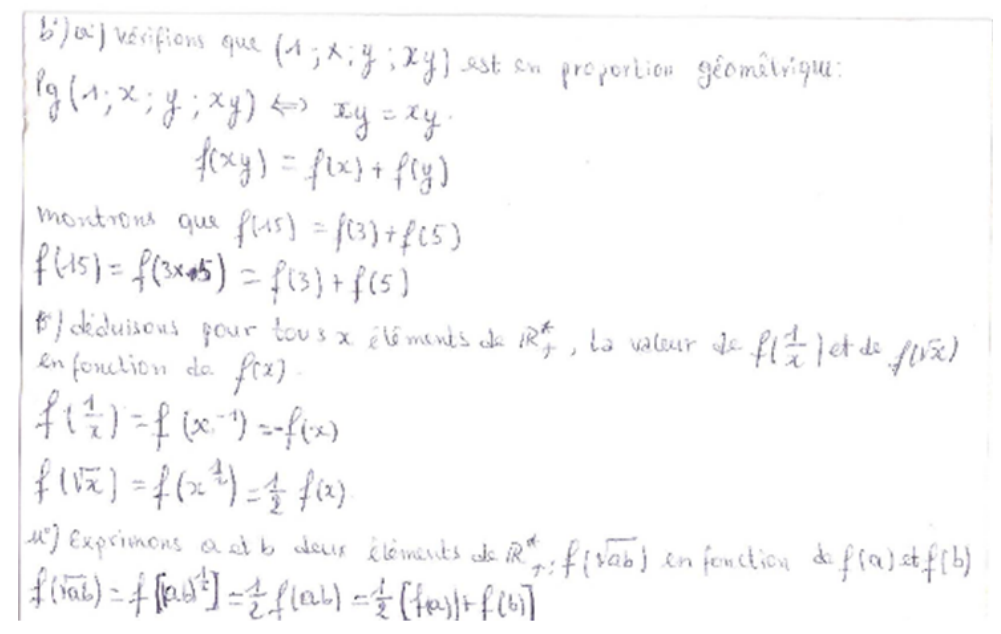

FIGURA 6 - RESOLUÇÃO APRESENTADA PELO GRUPO 8 (KONATÉ, 2006, p. 61).

Os alunos não tiveram qualquer problema ao preencher a tabela, nem na determinação da reta tangente à curva. É apresentado na Figura 7 um exemplo das produções dos alunos:

\section{Growits}

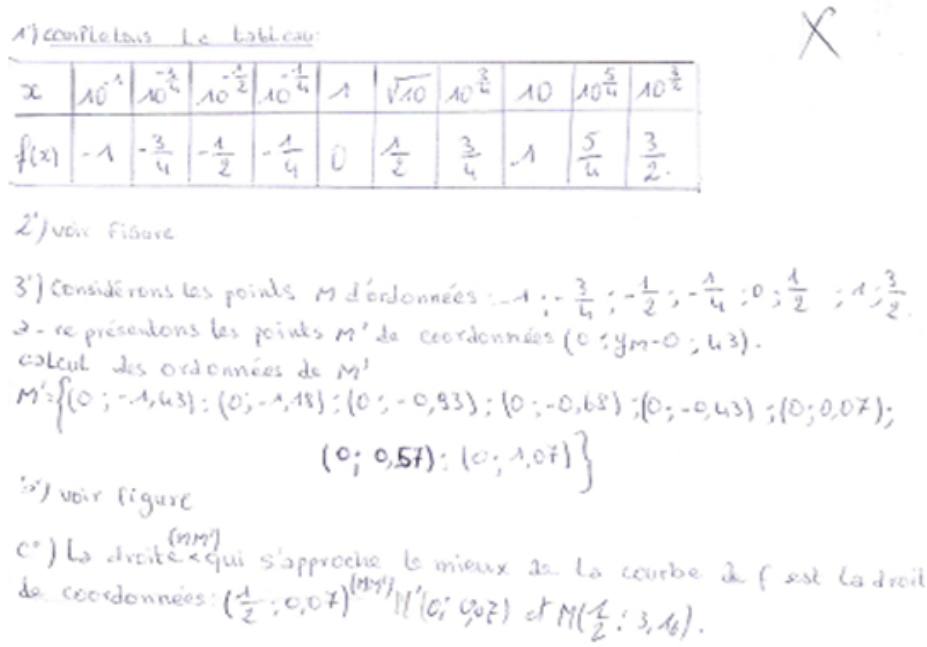

FIGURA 7 - RESOLUÇÃO APRESENTADA PELO GRUPO 8 (KONATÉ, 2006, p. 63). 


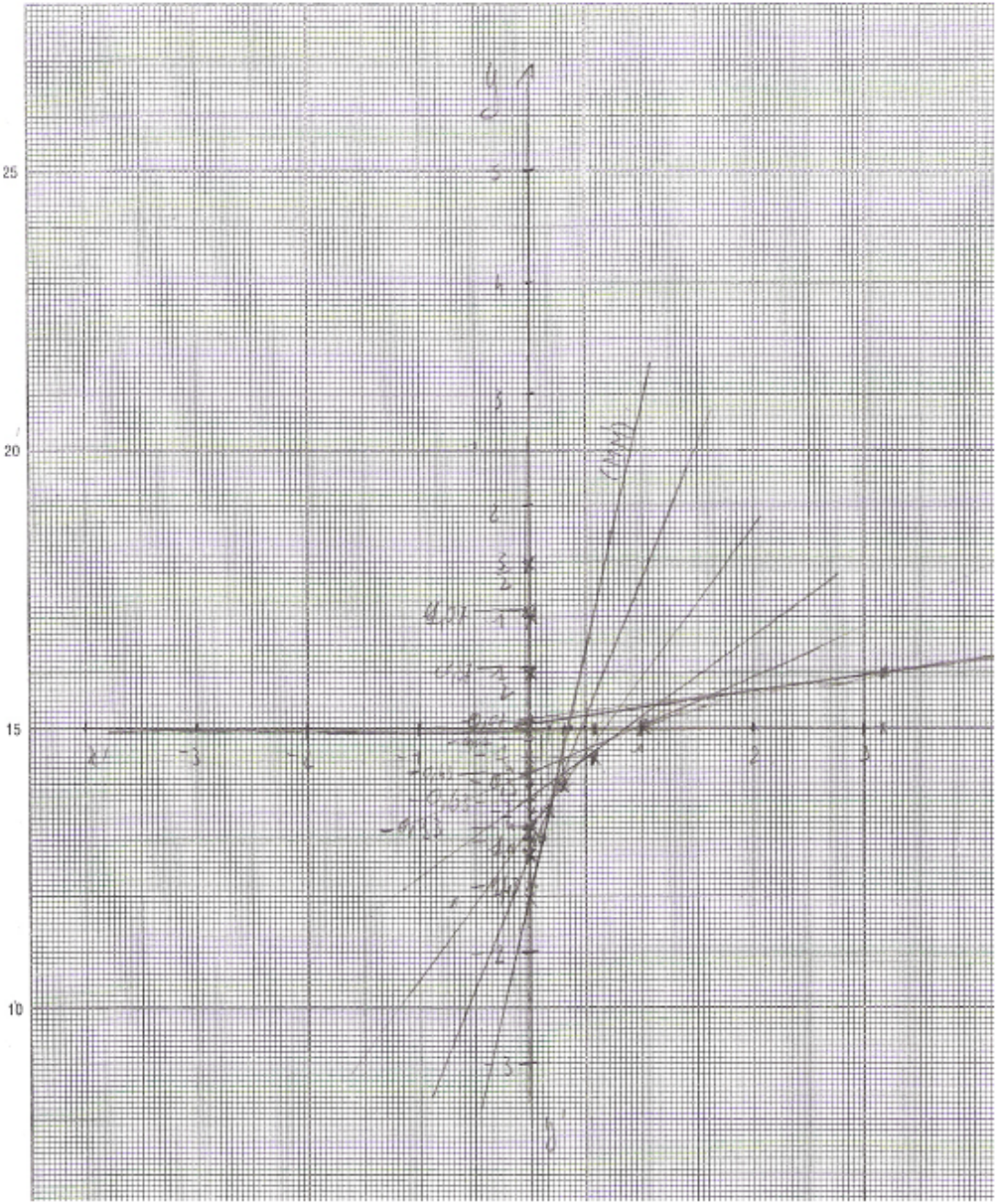

FIGURA 8 - PRODUÇÃO DO GRUPO 9 (KONATÉ, 2006, p. 64).

Com relação à representação gráfica da curva de f, o uso do papel milimetrado e a escolha de $2 \mathrm{~cm}$ com unidade nos eixos do plano cartesiano foram determinantes na construção do gráfico, como pode ser observado na produção do grupo 9 (Figuras 8, 9 e 10): 


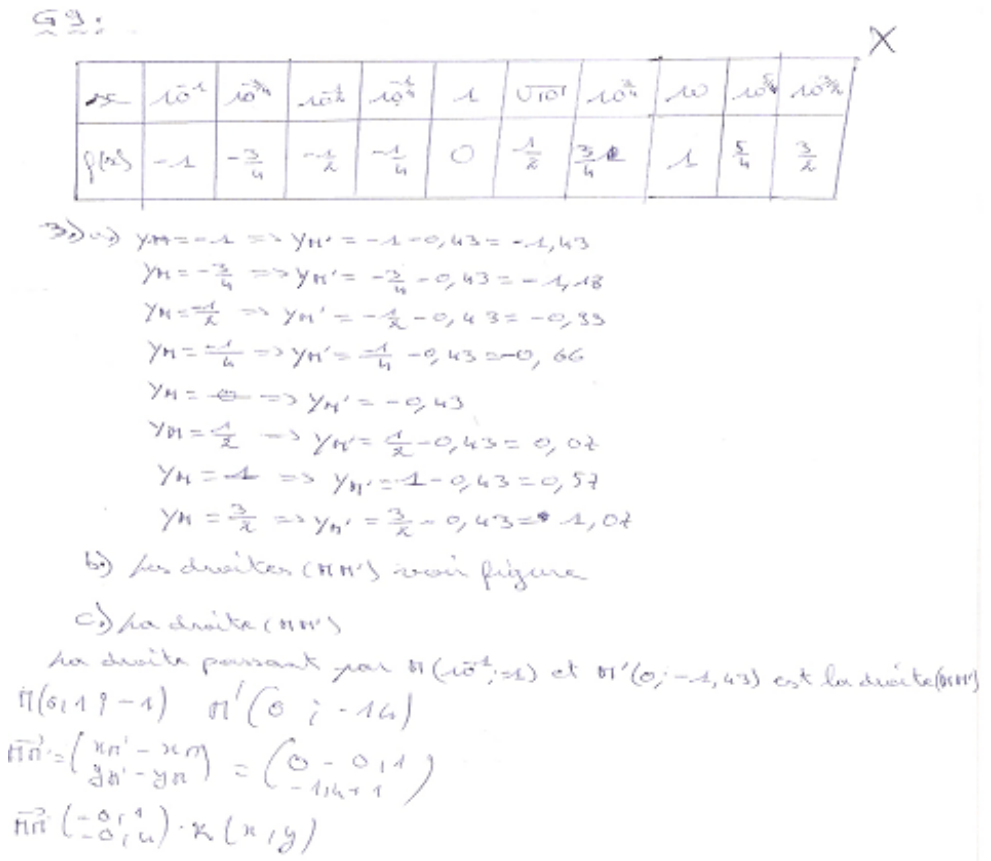

FIGURA 9 - PRODUÇÃO DO GRUPO 9 (KONATÉ, 2006, p. 65). 


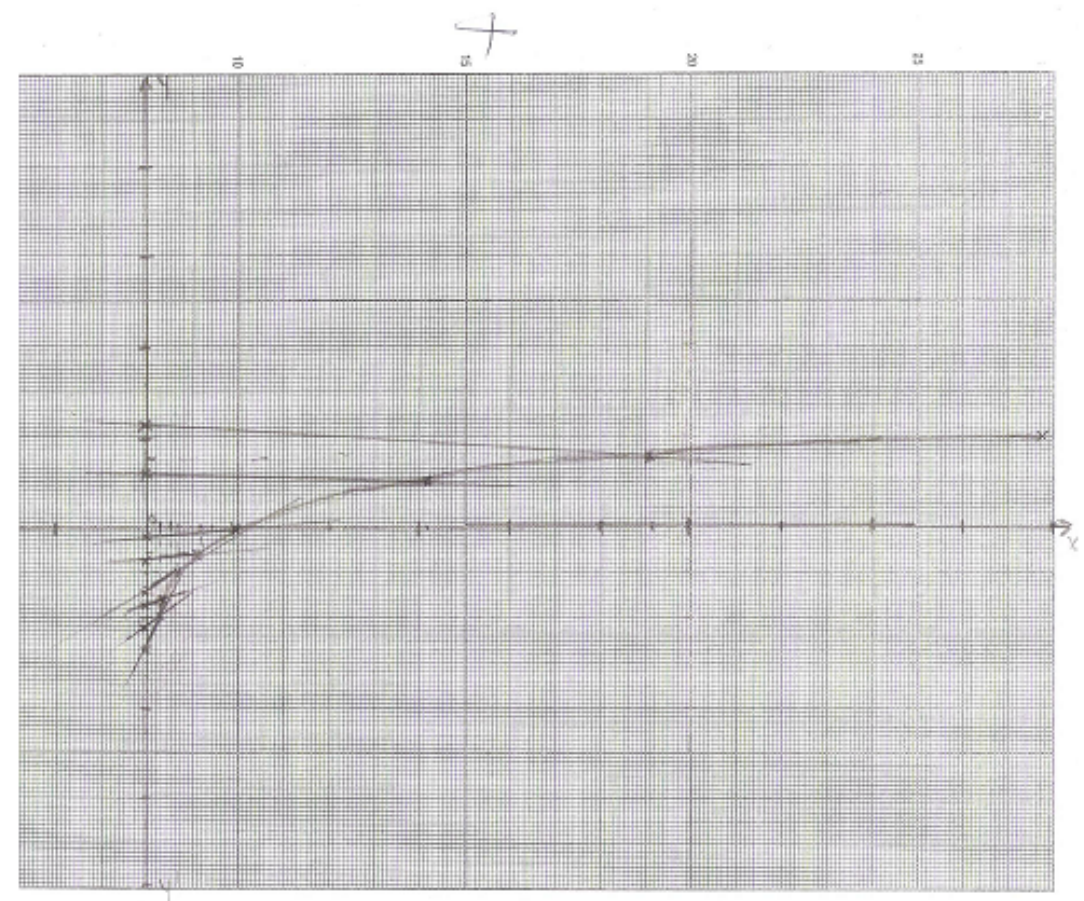

FIGURA 10 - PRODUÇÃO DO GRUPO 9 (KONATÉ, 2006, p 66).

\section{Conclusão}

Foi proposto, do ponto de vista da transposição didática, um método para introduzir e estudar o logaritmo a partir das noções de proporção aritmética e geométrica sem explicitar a lei da função. Além disso, conseguiu-se analisar determinados comportamentos dos estudantes, bem como as dificuldades que encontraram na sequência de Konaté (2006). Evidentemente, a situação-problema examinada não permite estudar todos os elementos do campo conceitual do logaritmo. Como pode ser percebido, ela focaliza o logaritmo de base decimal. Há, portanto, a necessidade de construção de outras situações cujo propósito seria o de estudar a função logaritmo de base diferente de 10. Além disso, há a necessidade de estudar a relação que existe entre a função logaritmo e a função exponencial. 


\section{REFERÊNCIAS}

ALMOULOUD, S. Fundamentos da didática da matemática. Curitiba: Editora da UFPR, 2007.

BROUSSEAU, G. Fondements et méthodes de la didactique des mathématiques. Recherche en didactique des mathématiques, Grenoble, v. 7, n. 2, p. 33-115, 1986.

CHEVALLARD, Y.; JOHSUA, M-A. La transposition didactique. Grenoble: La Pensée Sauvage-Éditions, 1991.

DOUADY, R. Jeux de cadres et dialectique outil-objet. Recherche en didactique des mathématiques, Grenoble, v. 7, n. 2, p. 5-31, 1986.

GROUPE MATH, IREM de Paris VII. Mathématiques: approche par des textes historiques. Repères-IREM, Pont-à-Mousson: Topiques Editions, v. 3, p. 43-52, 1991.

KONATE, S. L'enseignement et l'apprentissage des logarithmiques dans nos classes de terminales. Mémoire de DEA de Didactique des Mathématiques. Bamako- Mali: Université de Bamako, 2007.

VERRET, M. Le temps des études. Paris: Honoré Champion, 1975. 